\title{
ENERGY, TENSION AND FINITE TYPE MAPS
}

\author{
By Bang-Yen Chen, Jean-Marie Morvan \\ AND THÉRÈSE NORE
}

\begin{abstract}
We study the spectral geometry of smooth maps of a compact Riemannian manifold in a Euclidean space, by using the notion of order (introduced by the first author). We give some best possible estimates of energy and total tension of a map in terms of order. Some applications to closed curves and harmonic maps are then obtained. In the last section, we relate the spectral geometry of the Gauss map of a submanifold to its topology and derive some topological obstructions to submanifolds to have a Gauss map of low type.
\end{abstract}

\section{Introduction.}

Let $M^{n}$ be a compact Riemannian manifold and $x$ a smooth map from $M^{n}$ into the Euclidean space $E^{n+m}$. To study $x$, it is natural to consider the spectral decomposition of $x$ with respect to the Laplacian of $M^{n}$. This point of view has been adopted by the first author, when $x$ is an isometric immersion $[4,5]$. Using the same idea, we define two numbers $p$ and $q$, canonically associated with $x$; $p$ is a positive integer, and $q$ is either $\infty$ or an integer $\geqq p$. The pair $[p, q]$ is called the order of the map. A map is called a finite type map is $q$ is finite. Thus, we obtain spectral invariants related to the map. From Section 3 to Section 5, we relate the geometric properties of the map to its order and its type. In particular, we give in Section 3 a best possible estimate of the total tension of a map in terms of order, and then, in terms of $\lambda_{1}$ and energy. In Section 4, some relations between moment, energy and order are obtained. In Section 5 the notion of order is applied to obtain a necessary and sufficient condition for a spherical map to be harmonic. As an application of the previous sections, we study the Gauss map associated to a submanifold. We show that the spectral geometry of the Gauss map is related to the topology of the submanifold. In particular, if $M^{n}$ is a compact submanifold of $E^{m}$ with nonzero self-intersection number, the type of its Gauss map is "large" $(>n / 2)$.

The results of the first part of this paper have been announced in [6]. Some classifications of submanifolds with 1 or 2 type Gauss map can be found in [3], [7].

Received February 21, 1986 
A portion of this work was done while the first author was a visiting professor at the Universite d'Avignon, France. This paper was finished when the second and the third authors were visiting Michigan State University. The authors would like to thank their colleagues at both universities for their hospitality.

\section{Preliminaries.}

Let $M$ be a compact Riemannian manifold of dimension $n$ and $\Delta$ the Laplacian of $M$ acting on the space $C^{\infty}(M)$ of smooth functions. Then $\Delta$ has an infinite discrete sequence of eigenvalues:

$$
0=\lambda_{0}<\lambda_{1}<\lambda_{2}<\cdots<\lambda_{k}<\cdots \uparrow \infty .
$$

For each $k=0,1,2, \cdots$, the eigenspace $V_{k}=\left\{f \in C^{\infty}(M) \mid \Delta f=\lambda_{k} f\right\}$ is finitedimensional. With respect to the inner product $(f, g)=\int_{M} f g d V$ on $C^{\infty}(M)$, the decomposition $\Sigma_{k} V_{k}$ is orthogonal and dense in $C^{\infty}(M)$. Therefore, for each $f \in C^{\infty}(M), f=f_{0}+\sum_{t \geqq 1} f_{t}$, where $f_{0}$ is a constant and $f_{t}$ is the projection of $f$ into $V_{t}$.

For any smooth map $x: M \rightarrow E^{n+m}$ of the compact Riemannian manifold $M$ into the Euclidean $(n+m)$-space $E^{n+m}$, we can apply the above decomposition to the $E^{n+m}$-valued function $x$ :

$$
x=x_{0}+\sum_{t=1}^{\infty} x_{t},
$$

where $x_{0}$ is a constant vector and $x_{t}$ an eigenvector with $\Delta x_{t}=\lambda_{t} x_{t}$.

If $x$ is a non-constant map, there is a natural number $p$ such that $x_{p} \neq 0$ and $x=x_{0}+\sum_{t \geqq p} x_{t}$. If there are infinitely many nonzero $x_{t}$ 's in the decomposition (2.1), we put $q=\infty$. Otherwise, we put $q$ to be the largest integer such that $x_{q} \neq 0$ in the spectral decomposition (2.1). In any case, we have

$$
x=x_{0}+\sum_{t=p}^{q} x_{t}
$$

As in $[4,5]$, we call $[p, q]$ the order of the map $x$. Moreover, the map $x: M \rightarrow E^{n+m}$ is said to be of finite type if $q$ is finite. Otherwise, $x$ is said to be of infinite type. More precisely, $x$ is said to be of $k$-type $(k \in N \cup\{\infty\})$ if there exist exactly $k$ nonzero $x_{t}$ 's $(t \geqq 1)$ in the spectral decomposition (2.2).

If $M$ is a compact submanifold of $E^{n+m}$, then $M$ is a compact Riemannian manifold with respect to the induced Riemannian metric. In this case the submanifold $M$ is said to be of $k$-type if the immersion is of $k$-type.

The following result can be proved exactly in the same way as that of Theorem 2.1 of $[5$, p. 255]. (see, also [1]).

Proposition 2.1. Let $x: M \rightarrow E^{n+m}$ be a non-constant map of a compact 
Riemmanian manifold $M$ into $E^{n+m}$. Then $x$ is of finite type if and only if there is a non-trivial polynomial $Q(t)$ such that $Q(\Delta)\left(x-x_{0}\right)=0$.

It follows from (2.2) that $x_{0}=\int_{M} x d V / \int_{M} d V$, where $d V$ denotes the volume element of $M$. This simply says that $x_{0}$ is the center of mass of $x$.

If $\varphi: M \rightarrow N$ is a map between Riemannian manifolds, the energy-density $e(\varphi)$ of $\varphi$ is the real-valued function on $M$ given by

$$
e(\varphi)=\frac{1}{2}\|d \varphi\|^{2}=\frac{1}{2} \operatorname{trace}\left(\varphi^{*} g^{\prime}\right),
$$

where $g^{\prime}$ is the metric on $N$ and $d \varphi=\varphi^{*}$. The energy $E(\varphi)$ of $\varphi$ is defined by

$$
E(\varphi)=\int_{M} e(\varphi) d V
$$

The Euler-Lagrange operator associated with $E$ shell be written $\tau(\varphi)=\operatorname{div}(d \varphi)$ and called the tension field of $\varphi$. A map $\varphi$ is said to be harmonic if its tension field vanishes identically.

For the map $x: M \rightarrow E^{n+m}$, one has (cf. [8])

$$
\Delta x=-\tau(x) \text {. }
$$

Similar to Proposition 2.1, we have

PROPOSITION 2.2. Let $x: M \rightarrow E^{n+m}$ be a non-constant map of a compact Riemannian manifold $M$ into $E^{n+m}$. Then $x$ is of finite type if and only if there is a non-trivial polynomial $Q(t)$ such that $Q(\Delta) \tau=0$, where $\tau$ is the tension field of $x$.

If $x: M \rightarrow E^{n+m}$ is of finite type, there is a monic polynomial $P(t)$ of least degree with $P(\Delta) \tau=0$. The following result follows easily from Proposition 2.2 .

Proposition 2.3. If $x: M \rightarrow E^{n+m}$ is a finte type non-constant map, then

(1) the polynomial $P(t)$ is unique,

(2) if $Q$ is any polynomial with $Q(\Delta) \tau=0, P$ is a factor of $Q$, and

(3) $x$ is of $k$-type if and only if $k=\operatorname{deg} P$.

The same holds if $\tau$ is replaced by $x-x_{0}$.

The unique polynomial $P$, associated with the finite type map $x: M \rightarrow E^{n+m}$, is called the minimal polynomial of $x$.

If $x: M \rightarrow S_{c}^{n+m-1} \subset E^{n+m}$ is a map of $M$ into a hypersphere $S_{c}^{n+m-1}$ of $E^{m}$, then $x$ is called mass-symmetric if the center of mass, $x_{0}$, is the center $c$ of the hypersphere in $E^{n+m}$.

We shall make use of the following convention on the ranges of indices unless mentioned otherwise: 


$$
\begin{gathered}
1 \leqq i, j, k, \cdots \leqq n ; \quad n+1 \leqq r, s, t, \cdots \leqq n+m ; \\
n+1 \leqq \alpha, \beta, \gamma, \cdots \leqq n+m-1 .
\end{gathered}
$$

Remark 1. From (2.5) we know that if $x, \bar{x}: M \rightarrow E^{n+m}$ are two maps from a compact Riemannian manifold $M$ into $E^{n+m}$ such that $x, \bar{x}$ have the same tension field, then $x$ and $\bar{x}$ differ only by a translation.

\section{Total Tension and Order.}

Let $x: M \rightarrow E^{n+m}$ be a smooth map of a compact Riemannian $n$-manifold $M$ into $E^{n+m}$. Denote by $\tau=\tau(x)$ the tension field of $x$. The total tension $\mathscr{I}(x)$ of $x$ is given by

$$
\mathscr{I}(x)=\int_{M}\|\tau\|^{2} d V
$$

The following result gives a best possible estimate of the total tension in terms of the order.

THEOREM 3.1. Let $x: M \rightarrow E^{n+m}$ be a non-constant map of a compact Riemannian manifold $M$ into $E^{n+m}$. Then we have

$$
2 \lambda_{p} E(x) \leqq \int_{M}\|\tau\|^{2} d V \leqq 2 \lambda_{q} E(x),
$$

where $[p, q]$ is the order of $x$. Either equality sign in (3.2) holds if and only if $x$ is of 1-type.

Proof. Since $[p, q]$ is the order of $x$, we have

$$
x=x_{0}+\sum_{t=p}^{q} x_{t}
$$

Thus, by (2.5), we find

$$
-\tau=\sum_{t=p}^{q} \lambda_{t} x_{t}
$$

Since $\Delta=d \delta+\delta d$ is a self-adjoint operator on $C^{\infty}(M)$, we obtain from (2.3), (2.4) and (3.4) that

$$
\begin{gathered}
2 E(x)=\int_{M}\|d x\|^{2} d V=(d x, d x) \\
=(x, \Delta x)=\sum_{t=p}^{q} \lambda_{t}\left(x_{t}, x_{t}\right), \\
\int_{M}\|\tau\|^{2} d V=(\Delta x, \Delta x)=\sum_{t=p}^{q} \lambda_{t}^{2}\left(x_{t}, x_{t}\right) .
\end{gathered}
$$


Thus

$$
\int_{M}\|\tau\|^{2} d V-2 \lambda_{p} E(x)=\sum_{t=p}^{q} \lambda_{t}\left(\lambda_{t}-\lambda_{p}\right)\left(x_{t}, x_{t}\right) \geqq 0,
$$

equality holding if and only if $x_{p}$ is the only nonzero component. The other inequality is obtained in the same way.

(Q.E. D.)

If $x$ is an isometric immersion, Theorem 3.1 is due to [4].

The following corollaries follow immediately from Theorem 3.1.

COROLLARY 3.1. If $x: M \rightarrow E^{n+m}$ is a non-constant map of a compact Riemannian manifold into $E^{n+m}$, then we have

$$
\int_{M}\|\tau\|^{2} d V \geqq 2 \lambda_{1} E(x),
$$

equality holding if and only if $x$ is of order $[1,1]$.

If $x$ is an isometric immersion, (3.8) is due to [14].

CoRollary 3.2. Let $x: C \rightarrow E^{m+1}$ be a non-constant map of a closed curve into $E^{m+1}$. If $s$ denotes the arc length of $C$, then we have

$$
\int_{C}\left\|x^{\prime \prime}\right\|^{2} d s \geqq\left(\frac{2 \pi}{L}\right)^{2} \int_{C}\left\|x^{\prime}\right\|^{2} d s,
$$

where $L$ is the length of $C, x^{\prime}=d x / d s, x^{\prime \prime}=d^{2} x / d s^{2}$. Equality sign of (3.9) holds if and only if $x$ is of the form:

$$
x=c_{0}+c_{1} \cos \frac{2 \pi s}{L}+c_{2} \sin \frac{2 \pi s}{L},
$$

for some vectors $c_{0}, c_{1}, c_{2}$ in $E^{n+m}$.

This Corollary follows from the fact that the tension field of $x: C \rightarrow E^{n+m}$ is given !by $-x^{\prime \prime}$ and $\lambda_{1}$ of $C$ is equal to $(2 \pi / L)^{2}$ with the eigenspace $V_{1}$ spanned by $\cos (2 \pi s / L)$ and $\sin (2 \pi s / L)$.

By applying Corollary $3.2 k$ times, we obtain.

COROLlary 3.3. If $x: C \rightarrow E^{m+1}$ is a non-constant map of a closed curve $C$ in to $E^{m+1}$, then for any positive integers $k>h$, we have

$$
\int_{M}\left\|x^{(k)}\right\|^{2} d s \geqq\left(\frac{2 \pi}{L}\right)^{2 k-2 h} \int_{C}\left\|x^{(h)}\right\|^{2} d s,
$$

where $x^{(k)}=d^{k} x / d s^{k}$. The equality holds if and only if $x^{(h-1)}$ is of the form (3.10) for some vectors $c_{0}, c_{1}, c_{2}$ in $E^{m+1}$.

Remark 3.1. If $x: C \rightarrow E^{m+1}$ is an isometric immersion, inequality (3.9) 
reduces to

$$
\int_{C} \kappa^{2} d s \geqq \frac{4 \pi^{2}}{L},
$$

which is a variant of the famous Fenchel-Borsuk inequality, where $\kappa$ is the curvature of $C$ in $E^{m+1}$.

\section{Energy, Moment and Order.}

Now, we define the moment of a map as follows.

DEFINITION 4.1. Let $x: M \rightarrow E^{n+m}$ be a map of a compact Riemannian manifold $M$ into $E^{n+m}$ and $c$ a point in $E^{n+m}$. The moment of $x$ with respect to $c$ is defined by

$$
\mathscr{M}_{c}=\mathscr{M}_{c}(x)=\int_{M}\langle x-c, x-c\rangle d V .
$$

The moment of $x$ with respect to the center of mass $x_{0}$ is simply called the moment of the map $x$. We simply denote it by $\mathscr{M}$, i. e., $\mathscr{M}=\mathscr{M}_{x_{0}}$.

THEOREM 4.1. Let $x: M \rightarrow E^{n+m}$ be a non-constant map of a compact Riemannian manifold into $E^{n+m}$. Then we have

$$
\lambda_{p} \mathscr{M} \leqq 2 E(x) \leqq \lambda_{q} \mathscr{M} .
$$

Either equality sign of (4.2) holds if and only if $x$ us of 1-type.

Proof. Since $\Delta=d \delta+\delta d$, we have

$$
(x, \Delta x)=(x, \delta d x)=(d x, d x)=2 E(x) .
$$

From (3.3) we find

$$
(x, \Delta x)=\sum_{t=p}^{q} \lambda_{t}\left(x_{t}, x_{t}\right) .
$$

On the other hand, we have

$$
\mathscr{M}=\left(x-x_{0}, x-x_{0}\right)=\sum_{t=p}^{q}\left(x_{t}, x_{t}\right) .
$$

Therefore, (4.3), (4.4) and (4.5) imply

$$
2 E(x)-\lambda_{p} \mathscr{M}=\sum_{t=p}^{q}\left(\lambda_{t}-\lambda_{p}\right)\left(x_{t}, x_{t}\right) \geqq 0,
$$

equality holding if and only if $q=p$, i. e., $x$ is of 1-type. The other inequality is obtained in the same way. 
If $x$ is spherical, Theorem 4.1 yields the following best possible estimate of the energy.

COROLlARY 4.1. Let $x: M \rightarrow S^{n+m-1} \subset E^{n+m}$ be a mass-symmetric, non-constant map of a compact Riemannian manifold $M$ into a unit hypersphere $S^{n+m-1}$ of $E^{n+m}$. Then we have

$$
E(x) \geqq \frac{\lambda_{1}}{2} \operatorname{vol}(M) .
$$

Equality holds if and only if $x$ is of order $[1,1]$.

Proof. Under the hypothesis, we have $\mathscr{M}=\operatorname{vol}(M)$. Since $p \geqq 1$, (4.6) follows from (4.2). Equality sign of (4.6) holds if and only if $x$ is of 1-type with $p=1$.

(Q.E. D.)

For closed curves in $E^{m+1}$, Theorem 4.1 gives the following best possible estimate of moment.

Corollary 4.2. Let $C$ be a closed curve of length $L$ in $E^{m+1}$. Then the moment of $C$ satisfies

$$
\mathscr{M} \leqq L^{3} / 4 \pi^{2} .
$$

Equality holds if and only if $C$ is a plane circle of radius $L / 2 \pi$.

\section{Some Applications to Harmonic Maps.}

In this section we apply the notion of order to study harmonic maps.

LEMMA 5.1. Let $x: M \rightarrow S^{n+m-1} \subset E^{n+m}$ be a map of a compact Riemannian manifold $M$ into a hypersphere $S^{n+m-1}$ of $E^{n+m}$. Then the map $\bar{x}: M \rightarrow S^{n+m-1}$ is a harmonic map with positive constant energy density of and only if $x$ is a mass-symmetric, 1-type map.

Proof. Without loss of generality, we may assume that $S^{n+m-1}$ is a unit hypersphere centered at the origin of $E^{n+m}$. Denote by $j$ the inclusion of $S^{n+m-1}$ in $E^{n+m}$. Then the second fundamental forms $\sigma_{x}, \sigma_{\bar{x}}$ and $\sigma_{\jmath}$ of the maps $x, \bar{x}$ and $\jmath$ respectively satisfy

$$
\sigma_{x}(X, Y)=j_{*} \sigma_{\bar{x}}(X, Y)+\sigma_{j}\left(\bar{x}_{*} X, \bar{x}_{*} Y\right),
$$

for $X, Y$ tangent to $M$. Thus we have

$$
\Delta x=-\tau(x)=-\jmath_{*} \tau(\bar{x})-\sum_{\jmath=1}^{n} \sigma_{\jmath}\left(\bar{x}_{*} e_{\jmath}, \bar{x}_{*} e_{j}\right)
$$

where $e_{1}, \cdots, e_{n}$ is an orthonormal local frame on $M$. Since $\jmath$ is totally 
umbilical, (5.2) yields

$$
\Delta x=-\jmath_{*} \tau(\bar{x})+2 e(\bar{x}) x,
$$

where $e(\bar{x})$ is the energy density of $\bar{x}$.

If $x$ is a mass-symmetric 1-type map, we have $x=x_{p}$ and $\Delta x=\lambda_{p} x$, where $[p, p]$ is the order of $x$. Hence, (5.2) gives $\tau(\bar{x})=0$ and $e(\bar{x})=\lambda_{p} / 2$. Since $x$ is a non-constant map, $\lambda_{p}>0$. Thus, $\bar{x}$ is a harmonic map with constant energy density.

Conversely, if $\bar{x}$ is a harmonic map with constant energy density, then from (5.2) we find $\Delta x=2 e(\bar{x}) x$. This implies $x$ is a mass-symmetric, 1-type map.

(Q.E. D.)

By using Lemma 5.1 we have the following.

Proposition 5.1. Let $x^{\prime}:(M, g) \rightarrow S^{m+1}$ be a map from a Riemannan surface $(M, g)$ into $S^{m+1}\left(\subset E^{m+2}\right)$. If $x^{\prime}$ has positive energy-density $e^{\prime}=e\left(x^{\prime}\right)$, then $x^{\prime}$ is a harmonic map if and only if the composition:

$$
x:\left(M, e^{\prime} g\right) \stackrel{i d}{\longrightarrow}(M, g) \stackrel{x^{\prime}}{\longrightarrow} S^{m+1} \subset E^{m+2}
$$

is a mass-symmetric, 1-type map.

Proof. Let $e_{1}, \cdots, e_{n}$ be an orthonormal frame on $\left(M, e^{\prime} g\right)$. Then $\varepsilon e_{1}, \cdots, \varepsilon e_{n}$ $\left(\varepsilon=\sqrt{e^{\prime}}\right)$ is an orthonormal frame for $(M, g)$. Thus, the map $x:\left(M, e^{\prime} g\right) \rightarrow S^{m-1}$ has constant energy-density 1 . If $x^{\prime}:(M, g) \rightarrow S^{m+1}$ is harmonic, then it is known that the composition

$$
\left(M, e^{\prime} g\right) \stackrel{\imath d}{\longrightarrow}(M, g) \stackrel{x^{\prime}}{\longrightarrow} S^{m+1}
$$

is also harmonic (cf. [8]). Thus, by applying Lemma 5.1, we conclude that $x$ is a mass-symmetric, 1-type map.

Conversely, if $x$ is a mass-symmetric, 1-type map, then, by Lemma 5.1 , the composition :

$$
\left(M, e^{\prime} g\right) \stackrel{i d}{\longrightarrow}(M, g) \stackrel{x^{\prime}}{\longrightarrow} S^{m+1}
$$

is a harmonic map. Thus, $x^{\prime}=x^{\prime} \cdot i d \cdot \imath d^{-1}$ is also harmonic.

(Q. E. D.)

LEMma 5.1 implies immediately the following: A compact Riemannian manifold $M$ admits a harmonic map into a m-sphere with constant positive energydensity if and only if there is an eigenspace $V_{k}$ of $\Delta$ on $M$ which contains $m+1$ functions $f_{1}, \cdots, f_{m+1}$ with $f_{1}^{2}+\cdots+f_{m+1}^{2}=c$ for some nonzero constant $c$.

Remark 5.1. Some special cases of Proposition 5.1 were obtained in [12, 15]. 


\section{Topological Obstruction.}

Let $V$ be an oriented $m$-plane in $E^{n+m}$. Denote by $e_{n+1}, \cdots, e_{n+m}$ an oriented orthonormal basis of $V$. Then $e_{n+1} \wedge \cdots \wedge e_{n+m}$ is a decomposable $m$-vector of norm 1 and $e_{n+1} \wedge \cdots \wedge e_{n+m}$ gives the orientation of $V$. Conversely, a decomposable $m$-vector of norm 1 determines a unique oriented $m$-plane in $E^{n+m}$. Consequently, if we denote by $G(m, n)$ the Grassmannian of oriented $m$-planes in $E^{n+m}$, then $G(m, n)$ can be identified with decomposable $m$-vectors of norm 1. This shows that $G(m, n)$ can be regarded as an $n m$-dimensional submanifold of the unit hypersphere $S^{N-1}$ centered at the origin of $E^{N}=\Lambda^{m} E^{n+m}, N=\left(\begin{array}{c}n+m \\ m\end{array}\right)$ in a natural way. Thus, we have the following canonical inclusions:

$$
G(m, n) \subset S^{N-1} \subset E^{N}=\Lambda^{m} E^{n+m} .
$$

Let $x: M \rightarrow E^{n+m}$ be an isometric immersion of a compact oriented $n$-dimensional Riemannian manifold $M$ into $E^{n+m}$. For a vector $X$ tangent to $M$ we identify $X$ with its image under the differential $x *$ of $x$. If $e_{n+1}, \cdots, e_{n+m}$ is an oriented orthonormal normal frame on $M$, then the Gauss map $\nu$ :

$$
\nu: M \rightarrow G(m, n) \subset S^{N-1} \subset E^{N}=\Lambda^{m} E^{n+m}
$$

can be defined by $\nu(p)=\left(e_{n+1} \wedge \cdots \wedge e_{n+m}\right)(p)$.

The following result is known.

LEMMA 6.1. For a compact oriented submanifold $M$ in $E^{n+m}$, the Gauss map $\nu: M \rightarrow G(n, m) \subset S^{N-1}=E^{N}=\Lambda^{m} E^{n+m}$ is mass-symmetric in $S^{N-1}, N=\left(\begin{array}{c}n+m \\ n\end{array}\right)$.

Let $\nabla$ and $\nabla^{\prime}$ be the Levi-Civita connections of $M$ and $E^{n+m}$, respectively. Denote by $h, A$ and $D$ the second fundamental form, the Weingarten map and the normal connection of $M$ in $E^{n+m}$, respectively. For the second fundamental form $h$, we define the covariant derivative $\nabla h$ of $h$ by

$$
\left(\bar{\nabla}_{X} h\right)(Y, Z)=D_{X} h(Y, Z)-h\left(\nabla_{X} Y, Z\right)-h\left(Y, \nabla_{X} Z\right) .
$$

Let $\varphi: M \rightarrow N$ be a map between Riemannian manifolds. For vector fields $X, Y$ tangent to $M$, the symmetric bilinear map $\sigma: T M \times T M \rightarrow T N$ defined by

$$
\sigma(X, Y)=\bar{\nabla}_{X}^{\prime} f_{*} Y-f_{*} \nabla_{X} Y
$$

is called the second fundamental form of the map $\varphi$, where $\bar{\nabla}^{\prime}$ is the $\varphi$-induced connection on $\varphi^{-1}(T N)$.

In the following, we choose an oriented orthonormal local frame $e_{1}, \cdots, e_{n}$, $e_{n+1}, \cdots, e_{n+m}$ such that $e_{1}, \cdots, e_{n}$ is an oriented orthonormal local frame tangent to $M$ and $e_{n+1}, \cdots, e_{n+m}$ an oriented orthonormal local frame normal to $M$ in $E^{n+m}$. We denote by $h^{r}{ }_{\imath j}=\left\langle h\left(e_{\imath}, e_{j}\right), e_{r}\right\rangle$ the coefficients of $h$ and by $R^{D}$ the 
normal curvature tensor with coefficients $K_{s i j}^{r}=\left\langle R^{D}\left(e_{\imath}, e_{j}\right) e_{r}, e_{s}\right\rangle$. Then we have [3]

$$
\begin{aligned}
e_{i} \nu= & -\sum h^{r}{ }_{2 j} e_{n+1} \wedge \cdots \wedge e_{\jmath}^{r} \wedge \cdots \wedge e_{n+m}, \\
\Delta \nu= & \|h\|^{2} \nu+n \sum_{r} e_{n+1} \wedge \cdots \wedge \nabla H_{r} \wedge \cdots \wedge e_{n+m} \\
& -\sum_{r \neq s} \sum_{j<k} K^{r}{ }_{s j k} e_{n+1} \wedge \cdots \wedge e_{\jmath}^{r} \wedge \cdots \wedge e_{k}^{s} \wedge \cdots \wedge e_{n+m},
\end{aligned}
$$

where $H_{r}$ and $\nabla H_{r}$ denote the mean curvature and gradient of the mean curvature in the direction of $e_{r}$ and $e_{j}^{r}$ means to replace $e_{r}$ by $e_{\jmath}$.

By applying Proposition 2.3 we have the following.

THEOREM 6.1. Let $M$ be a compact oriented $n$-dimensional manifold immersed in $E^{n+m}$. If the Euler class $e\left(T^{\perp} M\right)$ of the normal bundle is nontrivial, then the Gauss map of $M$ in $E^{n+m}$ is of k-type with $k>m / 2$.

Proof. If either $m$ is odd or $m>n$, then the Euler class of normal bundle vanishes automatically. Thus we have $m \leqq n$ and $m=2 \delta$ is an even integer. by

For any positive integer $l \leqq m / 2$, let $V_{l}$ be the subspace of $\Lambda^{m} E^{n+m}$ spanned

$$
\left\{e_{\imath_{1}} \wedge \cdots \wedge e_{\imath_{2 l}} \wedge e_{r_{1}} \wedge \cdots \wedge e_{r_{m-2 l}}: 1 \leqq i_{1}, \cdots, i_{2 l} \leqq n, n+1 \leqq r_{1}, \cdots, r_{m-2 l} \leqq n+m\right\} .
$$

Denote by $\pi_{l}: \Lambda^{m} E^{n+m} \rightarrow V_{l}$ the canonical projection. Then from (6.5) we have

$$
\begin{gathered}
\pi_{\alpha}(\nu)=0, \quad \alpha \geqq 1, \\
\pi_{\alpha}(\Delta \nu)=0, \quad \alpha \geqq 2, \\
\pi_{1}(\Delta \nu)=-\sum K^{r}{ }_{s j k} e_{n+1} \wedge \cdots \wedge e_{\jmath}^{r} \wedge \cdots \wedge e_{k}^{s} \wedge \cdots \wedge e_{n+m} .
\end{gathered}
$$

Now, assume that the Gauss map $\nu$ is of $k$-type for some $k \leqq \delta$. Then, Proposition 2.3 and Lemma 6.1 imply that there is a monic polynomial $P$ of degree $k$ such that $P(\Delta) \nu=0$. Since $k \leqq \delta, Q(t)=t^{\delta-k} P(t)$ is a monic polynomial of degree $\delta$ such that $Q(\Delta) \nu=0$. In particular, we have

$$
\pi_{\delta}(Q(\Delta) \nu)=0 \text {. }
$$

By direct computation we have

$$
\pi_{\delta}\left(\Delta^{l} \nu\right)=0 \quad \text { for } l<\delta .
$$

Thus we have $\pi_{\delta}\left(\Delta^{q} \nu\right)=\pi_{\delta}(Q(\Delta) \nu)=0$. On the other hand, by direct computation, we may find

$$
\begin{aligned}
& \pi_{q}\left(\Delta^{\delta} \nu\right)=(-1)^{\delta} \sum K^{r_{1}{ }_{r_{2} \jmath_{1} \jmath_{2}}} \cdots K^{r_{m-1}}{ }_{r_{m} \jmath_{m-1} \jmath_{m}} \\
& e_{j_{1}}^{r_{1}} \wedge e_{j_{2}}^{r_{2}} \wedge \cdots \wedge e_{j_{m}}^{r_{m}}=0 .
\end{aligned}
$$


Thus we find

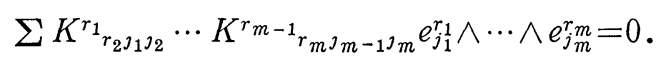

This is equivalent to

$$
\sum \varepsilon_{r_{1} \cdots r_{m}} K^{r_{1}}{ }_{r_{2} \jmath_{1} \jmath_{2}} \cdots K^{r_{m-1}}{ }_{r_{m} \jmath_{m-1} \jmath_{m}} \omega^{\jmath_{1}} \wedge \cdots \wedge \omega^{\jmath_{m}}=0,
$$

where $\omega^{1}, \cdots, \omega^{n}$ is the dual frame of $e_{1}, \cdots, e_{n}$ and $\varepsilon_{r_{1} \cdots r_{m}}$ is 1 or -1 according as $\left(r_{1}, \cdots, r_{m}\right)$ is an even or odd permutation of $(n+1, \cdots, n+m)$. Now, we put

$$
\Omega_{s}^{r}=\frac{1}{2} \Sigma K_{s i j}^{r} \omega^{2} \wedge \omega^{\jmath},
$$

where $\omega^{1}, \cdots, \omega^{n}$ is the dual frame of $e_{1}, \cdots, e_{n}$. Then (6.13) gives

$$
\gamma=\frac{(-1)^{\delta}}{2^{2 \delta} \pi^{\delta} \delta !} \sum \varepsilon_{r_{1} \cdots r_{2} \delta} \Omega_{r_{2}}^{r_{1}} \wedge \cdots \wedge \Omega_{r_{2 \delta}}^{r_{2} \delta-1}=0
$$

Since $\gamma$ represents the Euler class of the normal bundle, we obtain $e\left(T^{\perp} M\right)=0$.

(Q. E. D.)

For a compact, oriented $n$-dimensional submanifold $M$ immersed in $E^{2 n}$, the Euler number $\chi\left(T^{\perp} M\right)$ of the normal bundle is equal to twice of the self-intersection number [10]. Thus, from Theorem 6.1, we have the following.

COROLlaRy 6.1. Let $M$ be a compact, oriented, n-dimensional manifold $\imath \mathrm{m}$ mersed in $E^{2 n}$. If the self-intersection number of $M$ in $E^{2 n}$ is non-zero, then the Gauss map $\nu$ is of k-type with $k>n / 2$.

It is well-known that the self-intersection number is a regular homotopic invariant. From Theorem 6.1 we also have the following.

COROLLARY 6.2. Let $x: M \rightarrow E^{2 n}$ be an immersion of a compact, oriented, $n$-dimensional manifold $M$ in $E^{2 n}$. If the Euler class $e\left(T^{\perp} M\right)$ of the normal bundle of $x$ is nontrivial, then $x$ cannot be deformed regularly to an immersion with $k$-type Gauss map for $k \leqq n / 2$.

Example 6.1. Although the standard immersion of $S^{2 n}$ in $E^{2 n+1} \subset E^{4 n}$ has 1-type Gauss map, the Whitney immersion $w$ of $S^{2 n}$ in $E^{4 n}$ cannot be deformed regularly to an immersion with $k$-type Gauss map of $k \leqq n$. The Whitney immersion $w$ is defined as follows.

Let $f: E^{2 n+1} \rightarrow E^{4 n}$ be a map of $E^{2 n+1}$ into $E^{4 n}$ defined by

$$
f\left(x_{0}, x_{1}, \cdots, x_{2 n}\right)=\left(x_{1}, \cdots, x_{2 n}, 2 x_{0} x_{1}, \cdots, 2 x_{0} x_{2 n}\right) .
$$

Then $f$ induces an immersion $w: S^{2 n} \rightarrow E^{4 n}$, called the Whitney immersion, which has a unique self-intersection point $f(-1,0, \cdots, 0)=f(1,0, \cdots, 0)$. The self-intersection number $I(w)$ is one. Corollary 6.2 shows that $w$ cannot be 
deformed regularly to any immersion of $S^{2 n}$ in $E^{4 n}$ with $k$-type Gauss map for $k \leqq n / 2$.

If $x: M \rightarrow C^{n}$ is a totally real immersion, then the tangent bundle is isomorphic to the normal bundle. Thus, by Theorem 6.1 , we have the following.

COROLlary 6.3. Let $M$ be a compact, orented, $n$-dimensional, totally real submanifold of $\boldsymbol{C}^{n}$. If the Euler number $\chi(M)$ of $M$ is nontrivial, then the Gauss map of $M$ in $\boldsymbol{C}^{n}$ is of k-type with $k>n / 2$.

\section{REFERENCES}

[1] M. Barros and A. Ros, Spectral geometry of submanifolds, Note di Mat., 4 (1984), 1-56.

[2] M. Berger, P. Gauduchon And E. Mazet, Le spectre d'une variété Riemannienne, Lecture Notes in Math., 194, Springer-Verlag, 1971.

[3] D. D. BleEcker and J.L. Weiner, Extrinsic bounds on $\lambda_{1}$ of $\Delta$ on a compact manifold, Comm. Math. Helv., 51 (1976), 601-609.

[4] B.Y. CHEN, On the total curvature of immersed manifolds, IV, Bull. Math. Acad. Sinica, 7 (1979), 301-311; -, VI, ibid., 11 (1983), 309-328.

[5] B.Y. CHEN, Total mean curvature and submanifolds of finite type, World Scientific, 1984.

[6] B. Y. Chen, J.-M. Morvan and T. Nore, Energie, tension, et ordre des applications à valeurs dans un espace euclidien, C. R. Acad. Sc. Paris, 301 (1985), 123-126.

[7] B. Y. Chen And P. Piccinni, Submanifolds with finite type Gauss map, Bull. Austral. Math. Soc. (to appear).

[8] J. Eells and L. Lemaire, A report on harmonic maps, Bull. London Math. Soc., 10 (1978), 1-68.

[9] J. Eells and H. Sampson, Harmonic mappings of Riemannian manifolds, Amer. J. Math., 86 (1964), 109-160.

[10] R. Lashof and S. Smale, On the immersion of manifolds in Euclidean space, Ann. of Math., 68 (1958), 562-583.

[11] J. W. Milnor and J.D. Stasheff, Characteristic classes, Princeton Univ. Press, 1974.

[12] T.K. MiLnOR, The energy 1 metric on harmonically immersed surfaces, Michigan Math. J., 28 (1981), 341-346.

[13] T. NORE, Second fundamental form of a map, preprint, 1985.

[14] R.C. Reilly, On the first eigenvalue of the Laplacian for compact submanifolds of Euclidean space, Comm. Math. Helv., 52 (1977), 525-533.

[15] T. Takahashi, Minimal immersions of Riemannian manifolds, J. Math. Soc. Japan, 18 (1966), 380-385.

Department of Mathematics

Michigan State University

East Lansing, Michigan 48824, USA 
Faculté des Sciences d'Avignon

Departement de Mathematiques

33, RUe Louis Pasteur

84000 Avignon, France

Faculté des Sciences de Limoges

Departement de Mathematiques

123, RUe Albert Thomas

87100 LIMOGES, FRANCE 